\title{
Retroperitoneoscopic Standard or Hand-Assisted Versus Laparoscopic Standard or Hand-Assisted Donor Nephrectomy: A Systematic Review and the First Network Meta-Analysis
}

\author{
Paschalis Gavriilidis $^{\mathrm{a}, \mathrm{b}, \mathrm{c}}$, Vassilios Papalois ${ }^{\mathrm{b}}$
}

\begin{abstract}
Background: At the present four minimally invasive procedures namely retroperitoneoscopic (RPDN), laparoscopic (LPDN), handassisted retroperitoneoscopic (HARDN) and hand-assisted laparoscopic donor nephrectomy (HALDN) are used to perform donor nephrectomies. The current evidence based on retrospective studies and on pairwise only meta-analyses is inconclusive. Up to authors' best knowledge there is no so far network meta-analysis to compare all the above-mentioned procedures. Therefore, a network meta-analysis was conducted to compare the feasibility, safety and reproducibility of the four donor nephrectomies procedures.
\end{abstract}

Methods: Google Scholar, EMBASE, PubMed, and Cochrane library were used for a systematic literature search. Both updated pairwise and network meta-analyses were performed.

Results: Compared to LPDN there was evidence of significantly more right kidneys retrieved with RPDN; nonsignificant differences demonstrated both with HALDN and HARDN compared to LPDN. There was evidence that the operative time was significantly shorter by $77 \mathrm{~min}$ in RPDN compared to LPDN; on the other hand, HARDN and HALDN did not demonstrate significant differences when compared to LPDN.

Conclusions: The present study demonstrates that each approach can be applied safely in adequately selected patients. Moreover, retroperitoneoscopic is reliable, safe and easily reproducible alternative of LPDN for both left and right kidneys.

Keywords: Retroperitoneoscopic; Laparoscopic donor nephrectomy;

Manuscript submitted October 13, 2020, accepted October 27, 2020

Published online November 3, 2020

aDepartment of Vascular Access and Renal Transplantation, Queen Elizabeth University Hospitals Birmingham NHS Foundation Trust, B15 2TH, UK

${ }^{b}$ Department of Vascular Access and Renal Transplantation, Hammersmith Hospital, Imperial College Healthcare NHS Trust, London W12 0HS, UK

${ }^{\mathrm{c} C o r r e s p o n d i n g ~ A u t h o r: ~ P a s c h a l i s ~ G a v r i i l i d i s, ~ Q u e e n ~ E l i z a b e t h ~ U n i v e r s i t y ~}$ Hospitals Birmingham NHS Foundation Trust, Mindelsohn Way B15 2TH, UK.Email: pgavrielidis@yahoo.com

doi: https://doi.org/10.14740/jocmr4374
HALDN; HARDN; Safety

\section{Introduction}

The era of laparoscopic donor nephrectomy (LPDN) was inaugurated in 1995 by Ratner [1]. Afterwards, a randomized controlled trial (RCT) demonstrated that LPDN is associated with less pain, shorter recovery period and superior quality of life compared to open approach [2]. Consequently, individual studies and Cochrane review reported that LPDN is associated with major intraoperative complications that rarely happened with open approaches $[3,4]$. In order to avoid complications associated with challenging steps of the laparoscopic procedures some centers introduced and preferred the hand-assisted laparoscopic donor nephrectomy (HALDN) [5].

Furthermore, as an alternative to LPDN, the hand-assisted retroperitoneoscopic donor nephrectomy (HARDN) and retroperitoneoscopic donor nephrectomy (RPDN) were introduced, which combine the benefits of endoscopic procedure, handguided surgery, and the retroperitoneal access [3].

The existing evidence based on individual studies and on meta-analyses of a small number of patients is not strongly conclusive for the superiority of one approach over the other; and their results may had been influenced by institutional bias and underpowered samples. Up to authors' best knowledge there has been no network meta-analysis comparing LPDN, HALDN, RPDN and HARDN. Therefore, the first network meta-analysis was conducted to compare the feasibility, safety and reproducibility of the four minimally invasive donor nephrectomies.

\section{Materials and Methods}

The systematic search of the literature was conducted according to guidelines of the preferred reporting in systematic review and meta-analysis (PRISMA) checklist [6].

The Institutional Review Board (IRB) approval was not applicable; and the ethical compliance with human was not ap- 
plicable because this study does not contain any studies with human participants or animals performed by any of the author.

\section{Literature search}

EMBASE, MEDLINE (PubMed), Cochrane library, and Google Scholar databases were searched systematically using free text and MeSH terms (retroperitoneoscopic; transperitoneal; standard donor nephrectomy; living donor nephrectomy). Consequently, a search was conducted on www.clinicaltrials. gov to detect grey literature. Further search was conducted in the cited references of the retrieved articles to detect adequate articles. Any disagreements were resolved by consensus.

\section{Study, selection, and inclusion and exclusion criteria}

RCTs, retrospective studies, and case-matched studies that compared RPDN, LPDN, HALDN, and HARDN were included in this study.

\section{Data extraction and outcomes}

Two authors (PG and VP) independently detected the following parameters and data: age, body mass index (BMI), gender, right kidney retrieval, renal arteries more than one, cold ischemic time (CIT), warm ischemic time (WIT), operative time, estimated blood loss (EBL), delayed graft function, primary non-function, graft lost during the first month, creatinine, ureteral stricture, urine leak, renal artery stenosis, vein thrombosis, hospital length of stay; postoperative pain on day 1, 2, and 3 .

\section{Risk of bias assessment of included studies}

The validated Newcastle-Ottawa scale (NOS) was used to assess the methodological quality of the included studies; highquality studies were considered those that scored $\geq 7$ points [7].

\section{Statistical analysis}

First, a pairwise meta-analysis was conducted for studies that compared two of the laparoscopic donor nephrectomies approaches. Subsequently, a network meta-analysis was conducted to compare RPDN, LPDN, HALDN and HARDN. Statistical analysis was performed using General mixed treatments comparisons (GeMTC) software [8].

Hierarchical random-effects models were used to conduct network meta-analysis. Consequently, fixed-effects model was used and the results of the two models were compared to detect any discrepancy between them [9]. A fixed-effects model was also used to estimate whether any discrepancy could be detected between the results of the two models. The point-estimate was defined as the median of the posterior distribution based on 200,000 simulations; the corresponding $95 \%$ credible intervals (CrIs) were obtained using the 2.5th and 97.5th percentiles of the posterior distribution, which can be interpreted in a similar way as $95 \%$ confidence intervals (CIs) [10]. Heterogeneity and inconsistency of the indirect and direct evidence for the three surgical interventions were assessed by using the node splitting method [8-11].

Dichotomous variables were analyzed based on odds ratios (ORs) with a 95\% CI. For studies that did not report the means and variances for the two groups, these values were estimated from the median, range, and size of the sample, when possible, using the technique described by Hozo et al [12].

The significance level in all analyses was set at $\mathrm{P}<0.05$.

\section{Sensitivity analysis}

Both random and fixed effects models were used to assess the impact of heterogeneity on the results. Consequently, the results of the traditional and network meta-analysis were compared to detect any discrepancies between them.

\section{Results}

\section{Search strategy and included study characteristics}

Eight studies including 769 patients were selected from a pool of 97 studies (Fig. 1, Table 1 [13-19]). Of these patients, 403 $(53 \%)$ and $366(47 \%)$ underwent RPDN standard or hand-assisted and LPDN standard or hand-assisted, respectively [1319]. Three compared RPDN to HALDN [14-16], and three of HARDN vs. LPDN [13, 18, 19]. Ruszat et al [16] compared LPDN vs. HALDN vs. RPDN. Troppmann et al [17] compared HARDN to HALDN. The methodological quality of studies was high; all of them scored more than 7 (Table 1 [13-19]). Demographic characteristics demonstrated nonsignificant differences between the cohorts (Tables 1, 2 [13-19]).

\section{Statistically significant results of pairwise meta-analysis}

1) Right kidney retrieval

There was evidence that significantly more right kidneys retrieved in the RPDN standard and hand-assisted cohorts $(25 \%)$ compared to LDN standard and hand-assisted cohorts (4\%) $(\mathrm{OR}=5.43(1.10,26.67), \mathrm{P}=0.04)$.

\section{2) Operative time}

There was evidence that operative time was significantly shorter by 37 min in RPDN standard and hand-assisted cohorts compared to LDN standard and hand-assisted cohorts (mean difference $(\mathrm{MD})=-36.96(-54.68,-19.68), \mathrm{P}=0.01)($ Table 2 , $[13-19])$. 

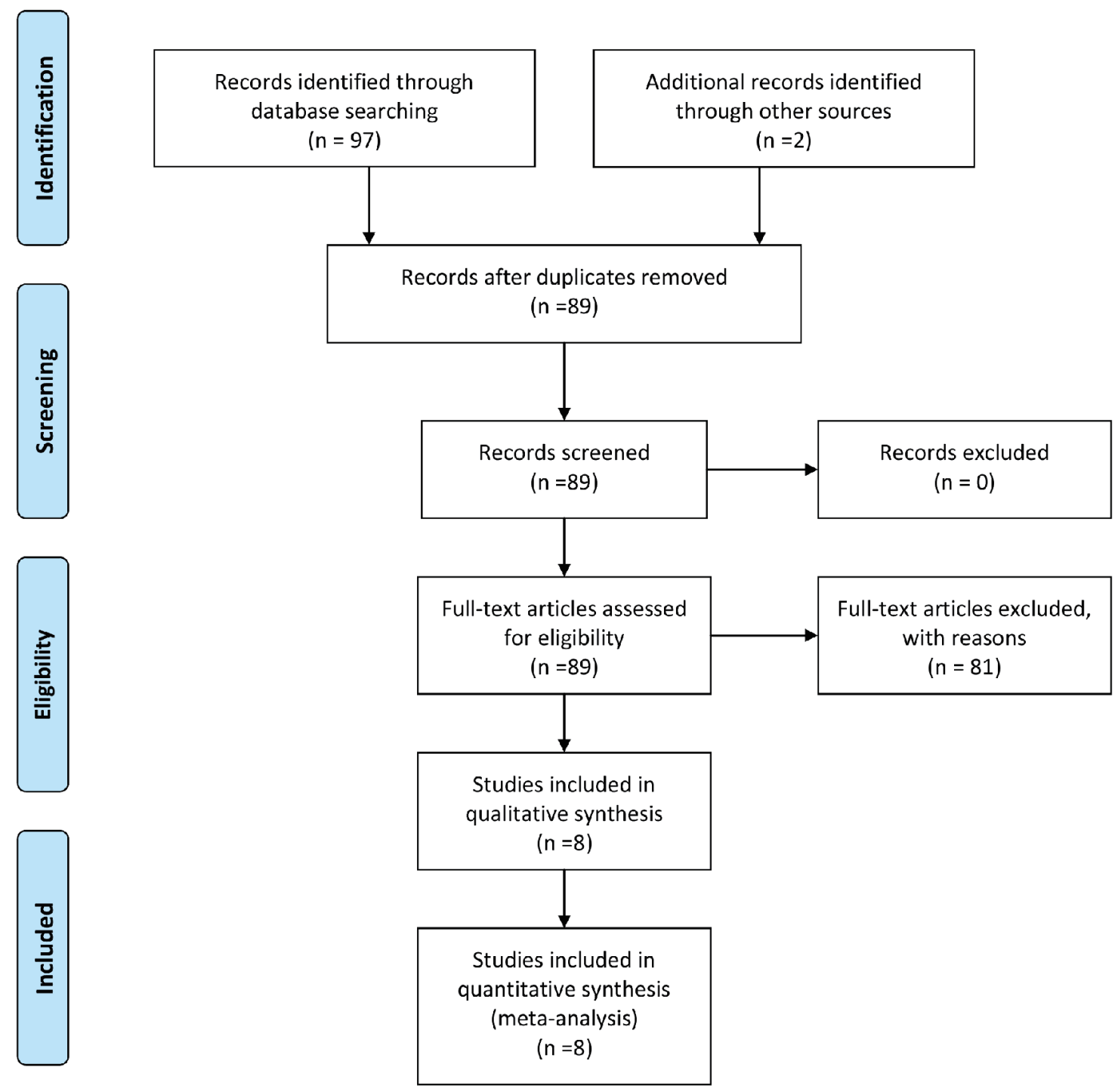

Figure 1. Diagram of the search strategy.

\section{Statistically nonsignificant results of pairwise meta-analysis}

Nonsignificant differences were detected in the variables of multiple arteries, conversion to open, EBL, WIT, intraoperative complications, postoperative graft complications, postoperative day 1-, 2-, 3- pain, and length of stay between the cohorts.

\section{Statistically significant results of network meta-analysis}

1) Operative time

There was evidence that RPDN and HARDN operative time was significantly shorter by 77 and 44 min compared to LPDN, (MD = -77 (CrI: -1.3, -22), MD = -44 (-86, -2.1)), respectively, (Fig. 2, Supplementary Material 1, www.jocmr. org).

\section{2) Right kidney retrieval}

There was evidence that significantly more right kidneys retrieved with RPDN procedure compared to LPDN (OR $=1.5$ (CrI: 47, 3.7). HALDN and HARDN demonstrated nonsignificant differences compared to LDN (Fig. 2)

\section{Statistically nonsignificant results on network meta-analysis}

There was evidence of nonsignificant differences in the variables of age, BMI, male gender, multiple arteries, EBL, WIT, length of stay, intraoperative and postoperative complica- 


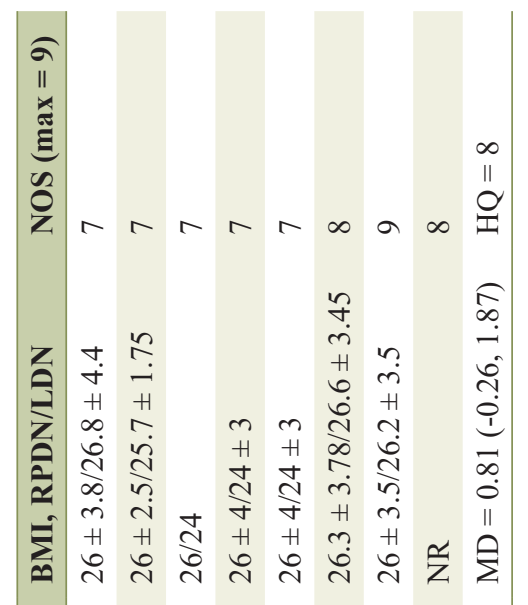

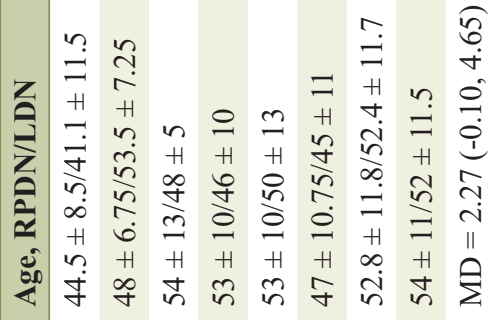

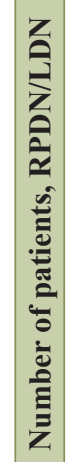

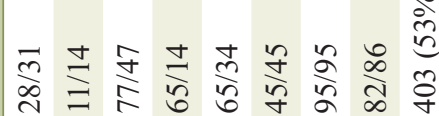
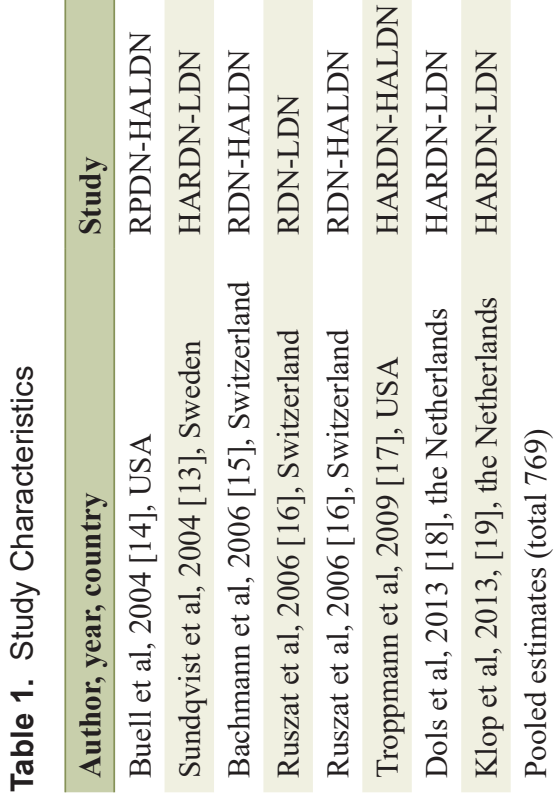

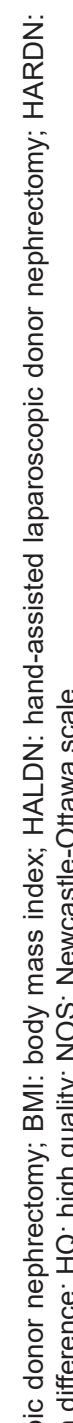

$\frac{\frac{\infty}{0}}{\frac{0}{\pi}}$

茪

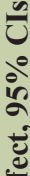

ì

กิ่

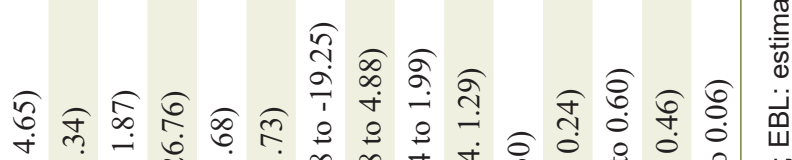

年

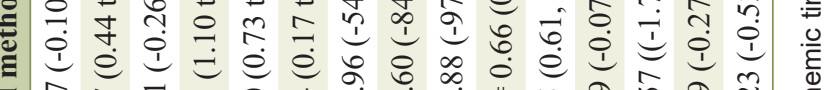

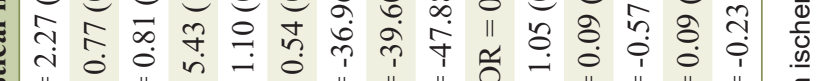

"

之
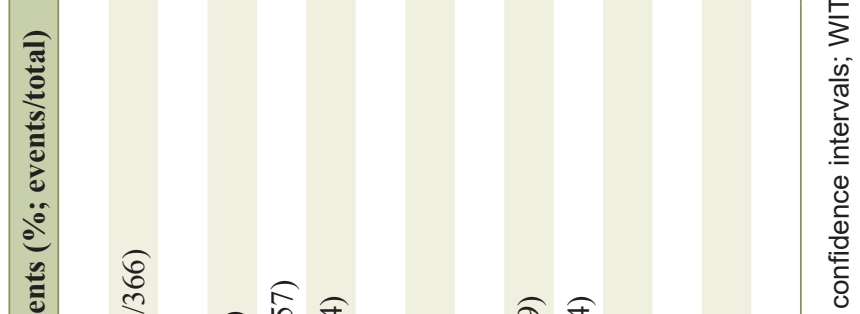

क

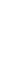

空

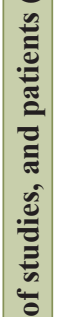

ఠి

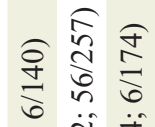

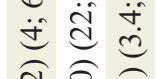

तิ

तु के हु

岂过

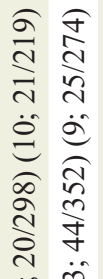

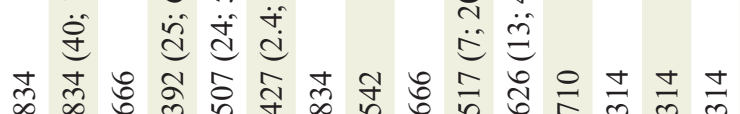

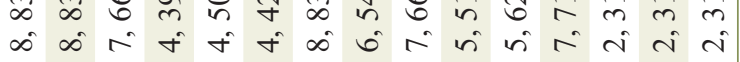

艺

हे

㐫 흥

융 음

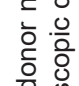

응

음

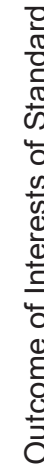

这变

zi

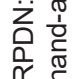

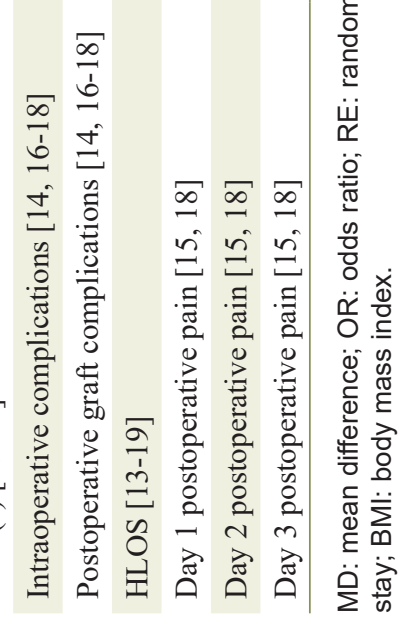


a

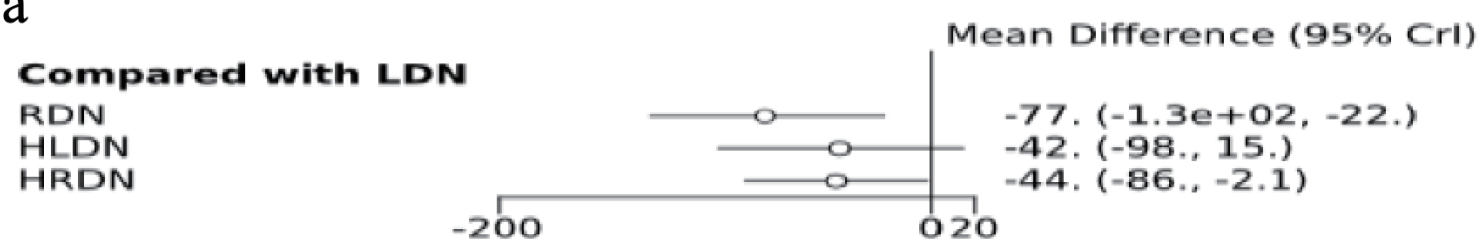

b

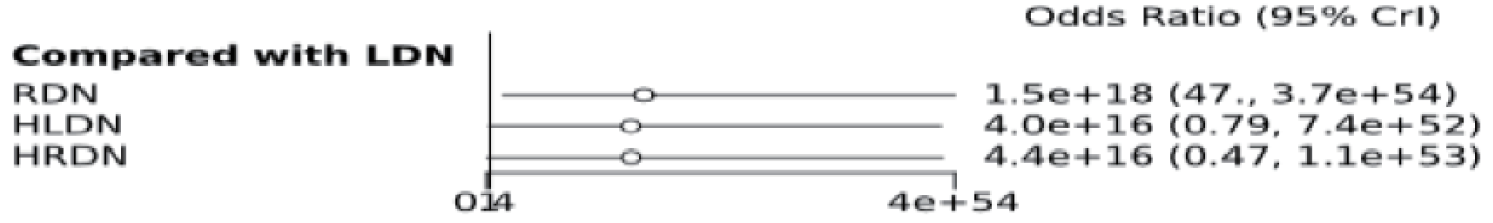

Figure 2. Forest plots of NMA depicting operative time and right kidney retrieval. (a) Operative time. (b) Right kidney retrieval.

tions between the cohorts (Supplementary Material 1, www. jocmr.org).

\section{Sensitivity analysis}

No discrepancies were detected between the results of the fixed- and random-effects models. In addition, no discrepancies were detected between the results of the pairwise and network meta-analysis. Moreover, network meta-analysis confirms that standard and hand-assisted RPDN demonstrate statistically significant shorter time compared to LPDN.

\section{Discussion}

The present study demonstrates that RPDN both its variations standard and hand-assisted demonstrated significantly shorter operative time compared to LPDN.

The shorter operative time can be explained with the obviating need to mobilize the colon and duodenum during the retroperitoneoscopic procedure [20]. Another reported privilege of retroperitoneoscopic procedure that may shorten the operative time is the rapid and easier access of the renal hilum allowing better exposure of the renal artery in a retrocaval location [14]. Another potential cause that would have influenced the operative time is that in many centers the retroperitoneoscopic procedure usually follows the transperitoneal [17]. Therefore, experienced laparoscopic operators will demonstrate shorter learning curve and better results.

Usually, the left kidney is preferred for transplantation because of its long vein. However, there is a consensus that the better kidney should remain with the donor [21]. In the present study, both standard and hand-assisted RPDN included significantly more right kidneys compared to LPDN.

It has been reported that BMI and gender may affect the level of complexity of the laparoscopic procedure [22]. In the present study, nonsignificant differences were detected between the cohorts.
It has been reported that intraoperative complications and especially bleeding is the principal cause for conversion to open [21]. In the present study, EBL, intraoperative complications demonstrated nonsignificant differences between the cohorts. Therefore, these findings translated to nonsignificant differences for conversion rate. Furthermore, WIT demonstrated nonsignificant differences between the cohorts and taking into account this finding with the above-mentioned could be explained the nonsignificant differences between the cohorts regarding the postoperative graft complications and consequently the nonsignificant differences in length of stay.

Two studies reported postoperative pain evaluation; nonsignificant differences were demonstrated between the cohorts.

Sensitivity analysis did not demonstrate any discrepancies between the fixed- and random-effects models. In addition, network meta-analysis confirmed the results of the traditional. However, the results of the present study should be interpreted in the context of its limitations. The majority of the studies were retrospective form single centers; therefore, national, institutional bias and underpowered sample may have influenced the results.

\section{Conclusions}

Up to authors' best knowledge this is the first network metaanalysis. It demonstrated nonsignificant differences regarding the variables of efficacy and safety between the four minimally invasive endoscopic procedures. Moreover, the shorter operative time of both standard and hand-assisted retroperitoneoscopic nephrectomy suggests easy reproducibility from surgeons experienced in LPDN. Therefore, each of the four procedures can be alternatively applied on adequately selected patients.

\section{Supplementary Material}

Suppl 1. Outcomes of interests of network meta-analysis of RDN, HRDN, LDN, and HLDN. 


\section{Acknowledgments}

None to declare.

\section{Financial Disclosure}

None to declare.

\section{Conflict of Interest}

All named authors hereby declare that they have no conflict of interest to disclose.

\section{Informed Consent}

Not applicable.

\section{Author Contributions}

Both authors contributed equally to the preparation and writing of the manuscript.

\section{Data Availability}

The authors declare that data supporting the findings of this study are available within the article.

\section{References}

1. Ratner LE, Ciseck LJ, Moore RG, Cigarroa FG, Kaufman HS, Kavoussi LR. Laparoscopic live donor nephrectomy. Transplantation. 1995;60(9):1047-1049.

2. Kok NF, Lind MY, Hansson BM, Pilzecker D, Mertens zur Borg IR, Knipscheer BC, Hazebroek EJ, et al. Comparison of laparoscopic and mini incision open donor nephrectomy: single blind, randomised controlled clinical trial. BMJ. 2006;333(7561):221.

3. Wilson CH, Sanni A, Rix DA, Soomro NA. Laparoscopic versus open nephrectomy for live kidney donors. Cochrane Database Syst Rev. 2011;11:CD006124.

4. Friedman AL, Peters TG, Jones KW, Boulware LE, Ratner LE. Fatal and nonfatal hemorrhagic complications of living kidney donation. Ann Surg. 2006;243(1):126130.

5. Wolf JS, Jr., Marcovich R, Merion RM, Konnak JW. Prospective, case matched comparison of hand assisted laparoscopic and open surgical live donor nephrectomy. J Urol. 2000;163(6):1650-1653.

6. Moher D, Liberati A, Tetzlaff J, Altman DG, The PRISMA Group . Preferred reporting items for systematic reviews and meta-analyses: the PRISMA statement. PLoS
Med. 2009;6(7):e1000097.

7. Wells GA, Shea B, O'Connell D, Peterson J, Welch V, Losos M, Tugwell P. The Newcastle-Ottawa scale (NOS) for assessing the quality of nonrandomised studies in meta-analyses. http://www.ohri.ca/programs/clinical_epidemiology/oxford.asp.

8. Van Valkenhoef G, Bujkiewicz S, Efthimiou O, Reid D, Stroomberg C, de Keijser J. GeMTC Manual. http:// www.gemtc.drugis.org.

9. Lu G, Ades AE. Combination of direct and indirect evidence in mixed treatment comparisons. Stat Med. 2004;23(20):3105-3124.

10. Ades AE, Sculpher M, Sutton A, Abrams K, Cooper N, Welton N, Lu G. Bayesian methods for evidence synthesis in cost-effectiveness analysis. Pharmacoeconomics. 2006;24(1):1-19.

11. Dias S, Sutton AJ, Welton NJ, Ades AE. Evidence synthesis for decision making 3: heterogeneity-subgroups, meta-regression, bias, and bias-adjustment. Med Decis Making. 2013;33(5):618-640.

12. Hozo SP, Djulbegovic B, Hozo I. Estimating the mean and variance from the median, range, and the size of a sample. BMC Med Res Methodol. 2005;5:13.

13. Sundqvist P, Feuk U, Haggman M, Persson AE, Stridsberg M, Wadstrom J. Hand-assisted retroperitoneoscopic live donor nephrectomy in comparison to open and laparoscopic procedures: a prospective study on donor morbidity and kidney function. Transplantation. 2004;78(1):147153.

14. Buell JF, Abreu SC, Hanaway MJ, Ng CS, Kaouk JH, Clippard M, Zaki S, et al. Right donor nephrectomy: a comparison of hand-assisted transperitoneal and retroperitoneal laparoscopic approaches. Transplantation. 2004;77(4):521-525.

15. Bachmann A, Wolff T, Giannini O, Dickenman M, Ruszat R, Gurke L, Kaufmann M, et al. How painful is donor nephrectomy? Retrospective analysis of early pain and pain management in open versus laparoscopic versus retroperitoneoscopic nephrectomy. Transplantation. 2006;81(12):1735-1738.

16. Ruszat R, Sulser T, Dickenmann M, Wolff T, Gurke L, Eugster T, Langer I, et al. Retroperitoneoscopic donor nephrectomy: donor outcome and complication rate in comparison with three different techniques. World J Urol. 2006;24(1):113-117.

17. Troppmann C, Daily MF, McVicar JP, Troppmann KM, Perez RV. The transition from laparoscopic to retroperitoneoscopic live donor nephrectomy: a matched pair pilot study. Transplantation. 2010;89(7):858-863.

18. Dols LF, Kok NF, d'Ancona FC, Klop KW, Tran TC, Langenhuijsen JF, Terkivatan T, et al. Randomized controlled trial comparing hand-assisted retroperitoneoscopic versus standard laparoscopic donor nephrectomy. Transplantation. 2014;97(2):161-167.

19. Klop KW, Kok NF, Dols LF, d'Ancona FC, Adang EM, Grutters JP, JN IJ. Cost-effectiveness of hand-assisted retroperitoneoscopic versus standard laparoscopic donor nephrectomy: a randomized study. Transplantation. 2013;96(2):170-175. 
20. Gill IS, Strzempkowski B, Kaouk J, et al. Prospective randomized comparison: transperitoneal vs retroperitoneal laparoscopic radical nephrectomy. J Urol. 2002;167:19.

21. Murray JE, Harrison JH. Surgical management of 50 patients with kidney transplant including 18 pairs of twins.
Am J Surg. 1963;105:205.

22. Miura M, Harada H, Fukuzawa N, et al. Approaches for laparoscopic live donor nephrectomy should be selected according to surgeon's skill and donor's physical status. Am J Transplant. 2009;9(Suppl 2):442. 